\title{
Information et communication : vers une éthique commune
}

Jean Devèze

\section{(2) OpenEdition}

12 Journals

Édition électronique

URL : http://journals.openedition.org/communicationorganisation/1807

DOI : 10.4000/communicationorganisation. 1807

ISSN : $1775-3546$

Éditeur

Presses universitaires de Bordeaux

Édition imprimée

Date de publication : 1 novembre 1995

ISSN : 1168-5549

\section{Référence électronique}

Jean Devèze, «Information et communication : vers une éthique commune », Communication et organisation [En ligne], 8| 1995, mis en ligne le 26 mars 2012, consulté le 02 mai 2019. URL : http:// journals.openedition.org/communicationorganisation/1807; DOI : 10.4000/ communicationorganisation. 1807

Ce document a été généré automatiquement le 2 mai 2019.

(c) Presses universitaires de Bordeaux 


\title{
Information et communication : vers une éthique commune
}

\author{
Jean Devèze
}

«On ne doit pas statuer par les lois divines ce qui doit être réglé par les lois humaines »(Montesquieu)

\section{L'éthique est-elle à l'ordre du jour?}

1 La responsabilité des communicateurs, en ces temps de diffusion massive de certaines informations, ou au contraire de secret soigneusement gardé sur d'autres, va en s'accroissant constamment. En est-il de même de l'esprit de responsabilité de ces mêmes communicateurs?

2 Divers événements récents permettent d'en douter et il y a lieu de s'interroger sur le développement de pratiques sans rapport avec les codes de déontologie, les chartes éthiques qui proscrivent le mensonge et prescrivent le respect de l'autre. Le fait est particulièrement marqué pour tout ce qui se rapporte à la communication internationale où les divergences d'intérêt, les tensions ou les conflits sont l'occasion de nombreuses manipulations de l'information et de la communication.

De nombreux observateurs, spécialistes ou non, ont abordé ces questions, l'un d'entre eux ${ }^{1}$ allant jusqu'à intituler un important chapitre de l'un de ses livres : « La communication sans éthique ni morale $»$.

4 Il constate que les médias, "cette puissance nouvelle semble très peu traversée par le débat moral, et ignore même le débat éthique que l'on revendique dans d'autres entreprises ${ }^{2} "$.

5 Rappelant que "la préoccupation de l'image pervertissait le plus souvent les déterminations morales des choix dans les entreprises", le même auteur enfonce vigoureusement le clou: "...dans le cas des médiateurs, à la fois diffuseurs et producteurs, l'acte même est dans la communication et ne peut s'en isoler. Or on peut être étonné de voir que le questionnement moral est presque absent de ce monde, sans conteste le plus cynique de notre société. La place et le 
pouvoir qu'il exerce dans cette société ne laissent donc pas d'inquiéter. On y entend évoquer des problèmes financiers, économiques, politiques, mais il est très rarement question de préoccupations morales $^{3} »$.

6 La préoccupation éthique est depuis déjà un certain temps présente au Canada et en particulier au Québec où des chercheurs comme Gilles Gauthier, Guy Giroux et Lise Boily ont publié de nombreux travaux dans la revue Communication ${ }^{4}$ ou dans la revue spécialisée, Cahiers de Recherche Éthique ${ }^{5}$, pilotés par des chercheurs de l'université du Québec à Rimouski.

\section{Quelle crise éthique?}

7 Le constat de ce fait ne dispense pas d'en rechercher les causes. L'analyse permet de repérer deux grands ordres de causes engendrant cet affaiblissement de la référence à un ensemble de valeurs jusqu'ici à peu près reconnues dans le monde de l'information et de la communication et, par voie de conséquence, les dérapages enregistrés.

8 Le premier est d'ordre économique et a déjà été largement évoqué par de nombreux auteurs américains et européens. Il s'agit de la recherche effrénée du gain d'argent, du profit immédiat, que l'on rencontre dans l'ensemble du monde capitaliste développé (mais aussi ailleurs...), donc dans celui des affaires en général et dans celui des affaires de presse, de publicité et de communication en particulier. Malgré son importance, nous ne nous y attarderons pas, car d'autres facteurs, plus méconnus, méritent de retenir ici l'attention.

9 Le deuxième ordre de causalité est d'ordre idéologique et se fonde sur la croyance aveugle qu'il n'existe qu'une seule vérité, comme l'enseignait la Logique d'Aristote, celle du tiers exclu. La conséquence immédiate en est que, chacun détenant sa vérité, - en tant qu'individu ou que membre d'une collectivité -, cette vérité-là est celle qu'il attend du communicateur s'il est en position de récepteur et celle qu'il sera incliné à proposer, voire à imposer, s'il est en position d'émetteur. La seconde conséquence, fréquemment observée, est une intolérance très forte à tout autre avis, opinion ou pensée que celle tenue pour être l'expression de la vraie vérité.

10 L'expérience de l'information en URSS de 1917 à 1990, celles des pays satellites, comme celle de la Chine et de nombreux autres pays a illustré jusqu'à la caricature cette dépendance de l'activité du communicateur vis-à-vis des valeurs de référence de l'idéologie officielle, communisme ou autre socialisme scientifique. Certains ont déduit de l'effondrement de ce système, et sans nul doute un peu trop hâtivement, que la communication entre les hommes, libérée de ce carcan idéologique, allait enfin baigner dans les eaux transparentes de la déesse Vérité, si nue et si belle.

11 C'est là négliger qu'il existe de par le monde d'autres systèmes prétendant détenir la Vérité vraie et unique, qu'il s'agisse de systèmes de croyance d'ordre religieux, économique, technique, etc. Tous caractérisés par l'affirmation de dogmes, assertions que le libre examen par la conscience humaine ne permet pas de classer en vraies ou fausses. Tout au plus apparaissent-elles incertaines ou indécidables.

12 Or dans les pays développés, l'information comme les moyens de la communication de masse s'adressent d'abord à des femmes et à des hommes que la complexité de la vie moderne interpelle, déstabilise et angoisse. Ceux-ci cherchent bien souvent la paix intérieure non en eux-mêmes (d'ailleurs le bruit des médias les en empêche), mais dans 
l'adhésion à des principes rassurants, c'est-à-dire assurant et réassurant, ceux-là même que véhiculent les dogmes.

\section{Quelques discours professionnels naïfs}

13 Quels sont les types de réponses observées, ça et là, aux questions soulevées par l'insuffisance éthique signalée de toutes parts?

14 On rencontre à ce sujet davantage d'intentions que de volontés, et aussi davantage de discours que de mises à l'épreuve. Aussi convient-il d'examiner attentivement certains textes anciens et certains débats récents. Les journalistes se réfèrent à ce propos à un texte de 1918, révisé et complété en 1938, connu sous le nom de «Charte des devoirs du journaliste », d'origine syndicale :

\section{Charte des devoirs du journaliste}

Déclaration de juillet 1918, révisée et complétée le 15 janvier 1938.

Un journaliste digne de ce nom prend la responsabilité de tous ses écrits, tient la calomnie, les accusations sans preuve, l'altération des documents, la déformation des faits, le mensonge, pour les plus graves fautes professionnelles; ne reconnait que la juridiction de ses pairs, souveraine en matière d'honneur professionnel; n'accepte que des missions compatibles avec sa dignité professionnelle; s'interdit d'évoquer un titre ou une qualité imaginaire, d'user de moyens déloyaux pour obtenir une information ou surprendre la bonne foi de quiconque; ne touche pas d'argent dans un service public ou une entreprise privée où sa qualité de journaliste, ses influences, ses relations soient susceptibles d'être exploitées; ne signe pas de son nom des articles de réclame commerciale ou financière; ne commet aucun plagiat, cite les confrères dont il reproduit un texte quelconque; ne sollicite pas la place d'un confrère ni ne provoque son renvoi en offrant de travailler à des conditions inférieures; garde le secret professionnel, n'use pas de la liberté de la presse dans une intention intéressée; revendique la liberté de publier honnêtement ses informations, tient le scrupule et le souci de justice pour des règles premières, ne confond pas son rôle avec celui du policier.

D'un point de vue juridique, ce texte n'engage que les adhérents des organisations syndicales qui le revendiquent: c'est par exemple le cas du Syndicat National des Journalistes en France, qui en signale "l'étonnante modernité " ${ }^{6}$ non sans avoir indiqué qu'il y a quelques années, "déontologie, éthique sonnaient comme autant d'académismes dépassés et ringards ${ }^{7} \%$.

Sous le titre équivoque "La déontologie à toutes les sauces", le rédacteur de cet article insiste sur la Charte européenne des journalistes, rédigée par un grand nombre de syndicats européens de professionnels avant de noter que l'essentiel est « de veiller à son application». Le Syndicat propose que cette charte soit inscrite dans la Convention collective des journalistes car cela «donnerait un fondement juridique, qui pour l'heure, est inexistant", à cette déontologie professionnelle. Il met l'accent sur le fait que «la déontologie se conjugue à la fois au singulier (le journaliste) et au Pluriel (sa rédaction)» car il résulte de la Charte que "le journaliste et sa rédaction engagent leur honneur professionnel personnellement vis-à-vis $d u$ public ${ }^{8} »$. Mais après avoir vivement proclamé qu'« il est temps de tordre le cou au vieux Projet d'instituer un Conseil de l'ordre de la presse ", suggéré.par de nombreux politiques ou spécialistes, le Syndicat National des Journalistes dégage en 
touche par une conclusion ambiguë : «C'est désormais à l'échelle européenne que ces questions doivent trouver leur réponse. » Lors de son 71e Congrès à Nogent-le Rotrou en octobre 1991, ce même Syndicat proclame en son communiqué final :

"Le Congrès du Syndicat National des journalistes a consacré une large part de ses travaux

aux problèmes liés à la déontologie professionnelle»

Depuis sa création, le SNJ a agi souvent seul pour que la profession de journaliste s'entoure de références éthiques. C'est lui qui est à l'origine des textes majeurs de notre profession (Charte des devoirs, loi de 1935 sur le statut du journaliste). Jusqu'à présent, les employeurs se sont constamment refusés à inclure la Charte dans la convention collective.

Les écoles de journalisme ont un rôle primordial à jouer dans la compréhension et la diffusion de nos principes professionnels.

Mais c'est d'abord aux lecteurs, aux auditeurs et téléspectateurs que s'adresse le message du SNJ à l'issue de son congrès. Nous voulons engager avec eux une large réflexion sur le rôle essentiel d'une presse libre, responsable et pluraliste pour assurer l'expression démocratique.

Le Congrès appelle l'ensemble de ses adhérents à engager auprès des enseignants, des élus, des associations, des actions de sensibilisation aux réflexions du SNJ et de les y associer.

(...) Le Congrès mandate la Commission (de déontologie) pour poursuivre et approfondir sa réflexion, notamment sur les moyens pratiques de faire appliquer et respecter notre déontologie. "

Outre le fait que les engagements pris dans ce texte ne semblent nullement avoir été tenus (où en est la large réflexion avec les lecteurs, auditeurs, etc., annoncée ?), on ne peut manquer d'être frappé par le caractère naïf de telles propositions, qui paraissent « détachées » de leurs contextes institutionnels, économiques, politiques et sociaux.

\section{La réflexion du monde catholique}

C'est de ce côté que l'on rencontre, en matière d'information et de communication, la réflexion la plus ample, la plus profonde et la plus diversifiée.

En témoignent ainsi les travaux conduits par le P. C.-J. Pinto de Oliveira, dominicain enseignant l'éthique de la communication à l'Institut de journalisme et des communications sociales de l'Université de Fribourg en Suisse ${ }^{9}$; la tenue, en mars 1991, d'un colloque "Chrétiens professionnels de la communication" à Angers ${ }^{10}$ avec une brochette de journalistes catholiques et le cardinal Poupard, président du Conseil pontifical de la culture; de même la réunion (mars 1992) au Centre Thomas More, à l'abbaye dominicaine de l'Arbresle d'un colloque intitulé : «Pratiques des journalistes, éthique et réflexion chrétienne ».

La pensée des catholiques y apparaît dans sa plus grande diversité, de la plus traditionnelle et la plus conservatrice jusqu'à la plus moderniste, voire "progressiste ", que ce soit sur le rôle des médias, la question de la vérité ou de l'objectivité, la relation à la foi et à l'Église catholique ou le programme de «nouvelle évangélisation » de l'actuel pontife Jean-Paul II.

Pour en rester seulement aux questions d'éthique de la communication, on rencontre généralement l'opinion selon laquelle la liberté (de l'information, de la communication) est étroitement liée à la responsabilité.

Ainsi Philippe Guilhaume, après avoir stigmatisé «les indignations et les condamnations", note-t-il : 
30 Chacun connait le risque que soit considérée comme une bonne information l'information élogieuse, celle qui présente de façon positive les thèses auxquelles on souscrit. De même, je crois qu'il faut se méfier des condamnations globales. Refuser que soient condamnés des informations, des informateurs ou les médias. Même si cette condamnation est prononcée par le Pape. (...) Il y a les solutions corporatistes ou réglementaires. Je crois qu'il faut se féliciter du rôle de réflexion collégiale que tiennent, de plus en plus dans l'ensemble les conférences de rédaction. Mais je pense qu'il faut se méfier des solutions corporatistes qui me paraissent être une grave récession.

31 Se méfier de ceux qui suggèrent la constitution d'un Ordre des journalistes. De même qu'il faut se méfier de toutes les tentations de réglementation, de législation supplémentaire, au seul prétexte que moins on organise les libertés, mieux elles se portent. Le seul corollaire acceptable à la liberté c'est la responsabilité, le seul corollaire acceptable à la liberté de linformation c'est la responsabilité individuelle des journalistes et la responsabilité concertée des équipes de rédacteurs et des éditeurs ${ }^{11}$."

Gustave Thibon rappelle d'abord l'importance, capitale à ses yeux, de la communication, avant de s'interroger sur la « quête du sens »:

"La communication a une importance capitale dans la vie des hommes en général, dans tous les temps et dans tous les lieux. L'homme, disait Aristote, est un animal politique, social, et donc il n'acquiert sa vraie stature d'homme qu'au travers de la communication, qu'à travers ce que lui ont transmis les autres hommes. C'est une chose évidente: l'homme se tient à l'écart des animaux en général, qui communiquent entre eux, mais qui sont largement " programmés » par la nature. L'homme, sans transmission d'une culture qui le nourrit, sans communication, n'arrive pas à être un homme ${ }^{12}$."

«Devant la masse d'informations, de désinformations, d'informations contradictoires, qui nous menace d'abrutissement, je crois qu'il y a une nécessité impérieuse du retour de l'homme sur lui-même, de réflexions, d'attention extrême. Trop souvent les moyens de communication ne favorisent pas l'attention, au contraire un clou chasse l'autre. On parle de distraction; or, être distrait, ce n'est pas bon (étymologiquement, cela veut dire: "tirer dehors et de travers $")^{13}$."

\section{Il converge en cela avec Patrick Chalmel :}

«Les hommes eux communiquent, et ils communiquent pour communier. D'ailleurs, même lorsqu'ils n'ont pas le prétexte d'informations à échanger, ils s'arrangent quand même pour communiquer dans le but d'une certaine communion. On dit qu'ils parlent alors «de la pluie et du beau temps ${ }^{14}$ ». La communication, assure-t-il, est « affectée par la perte du sens »: «Première de ces maladies de la communication, la perte du sens des mots et de la précision des concepts ${ }^{15}$ "

Elle souffre également de la «maladie du doute universel »: "Parmi les maladies graves qui affectent aujourd'hui la communication, je placerais également le doute excessif ${ }^{16}$."

(...) «On note ainsi aujourd'hui dans de nombreux domaines l'envahissement de la raison par un doute excessif, non fondé, symptôme d'intelligences flottantes, découragées, qui trouble et vide de son sens la communication ${ }^{17}$."

Troisième affection diagnostiquée par le journaliste de « Famille chrétienne », la crédulité absolue :

"Tout aussi grave et plus voyante encore, la crédulité délirante de nos contemporains, qui pour avoir douté des vérités de bon sens au nom d'une rationalité supérieure, sont aujourd'hui tombés dans l'irrationnel le plus aberrant ${ }^{18}$."

Patrice de Plunkett met en cause l'idéologie implicite des médias qui conduit à une vision du monde très réductrice : 

puissance et en extension ${ }^{24}$."

51 Sur la question de la vérité, le P. Pinto de Oliviera tient un discours éthique qui ne s'appuie sur aucun des dogmes de l'Église. Il constate ainsi la difficulté attachée à la "notion même de vérité reléguée parmi les idées imprécises et inopérantes. Surtout ne parlons pas d'objectivité puisque " tout est subjectif» dans la captation et la transcription des nouvelles. La première dépêche ne serait-elle pas déjà le résultat de la première interprétation d'une agence qu'un réseau plus ou moins long d'interprétations transmettra à l'interprétation du journaliste qui la confiera à l'interprétation du lecteur... 


$$
\begin{aligned}
& \text { d'interprétation, d'analyse, de confrontation - à la saisie d'une réalité elle-même complexe, souvent } \\
& \text { changeante lorsqu'il s'agit de la réalité sociale }{ }^{25} \text {. " } \\
& \text { "Paradoxalement, la subjectivité authentique - celle qui va jusqu'au bout de ses exigences - } \\
& \text { comporte déjà une certaine objectivité. Elle est une forme de vérité se faisant jour dans } \\
& \text { l'intimité de l'esprit, tendant à s'affirmer comme conscience en harmonie avec la "réalité » } \\
& \text { des sentiments et leur correspondance avec la situation telle que la perçoit l'intelligence ". } \\
& \text { "La vérité n'est pas impossible. Elle est rudement difficile-Pas de vérité toute donnée. Elle } \\
& \text { exige un travail de vérification. De chaînon en chaînon, à travers le long processus de la } \\
& \text { communication, la nouvelle est menacée de déformation. Le journaliste aura à se faire } \\
& \text { critique, vérificateur"7 » } \\
& \text { "Tel est le sens et telle est surtout l'extrême exigence de la vérité comme valeur primordiale } \\
& \text { de l'information.» (...) } \\
& \text { "La communication est un système en symbiose avec d'autres systèmes: socio politiques, } \\
& \text { économiques, culturels. »...) } \\
& \text { "Le système de communication et d'information de lui-même pousse à occulter les } \\
& \text { mécanismes et les voies de son fonctionnement. Le journaliste aura à assumer les fonctions } \\
& \text { de détective ou de thérapeute pour arracher des bribes de vérité à un système social et } \\
& \text { communicationnel inexorablement pervers et perverti!» (...) }
\end{aligned}
$$

vérité s'accomplit comme perfection vivante de l'intelligence qui s'ouvre - par un processus

54 " Mais en tout état de cause, en professant que la vérité est la valeur primordiale de l'information, on n'en fera pas une oasis tout confort, tout repos. Elle se dressera toujours comme un sommet âpre et exaltant à gravir. Le savant, l'historien, le journaliste sont payés pour le savoir. Souvent, ils auront plutôt à payer de leurs personnes ${ }^{28}$. »

55 Ce point de vue est largement partagé par Philippe Guilhaume, l'ancien Président d'Antenne 2, qui cite d'abord Etienne Mougeotte, aujourd'hui directeur à TF1 : "Il ne suffit pas de diffuser une interview ou des images, il faut encore en préciser les conditions de tournage, analyser les intentions de ceux qui ont laissé filmer ou se sont laissé interviewer. "

Et de recommander la "traque de la vérité »: "Or la vérité se cache, la vérité dérange, la vérité se vend mal et la vérité est inconfortable ${ }^{29}$."

58 Avant d'entreprendre l'analyse des causes pour lesquelles il en est ainsi et de poser cette redoutable question : "Est-ce que le citoyen est capable d'entendre la vérité ? " 30

Les remèdes qu'il propose sous le nom de «solutions » ne font appel à aucune référence dogmatique : «Alors, est-ce qu'il y a des solutions? «

La première solution, c'est de faire confiance aux hommes et d'imaginer que la réflexion éthique ne sera pas seulement un objet de colloque, mais sera une interrogation permanente des individus distributeurs ou consommateurs d'informations. On peut faire ça. On peut faire confiance.

61 Il y a une deuxième solution qui est d'essayer d'organiser finalement des prétextes à la véracité de l'information. Il y a deux choses qui me paraissent intéressantes. Certains journaux anglo-saxons développent le sens critique de leurs lecteurs en publiant en encadré, en première page, toutes les semaines, la liste des erreurs d'information qu'ils ont commises la semaine précédente. Ce n'est pas inintéressant, ça développe nécessairement le sens critique.

62 Il y a des radios et des télévisions Scandinaves et canadiennes qui ont créé un "ombudsman", c'est-à-dire un homme sans fonction hiérarchique auquel les journalistes peuvent s'adresser pour 
demander avis, conseil, et protection contre d'éventuelles pressions. On peut, peut-être, imaginer d'autres solutions.

63 Moi, je crois profondément que la solution la plus efficace c'est, d'une part la formation des journalistes et de tous les hommes de communication, la formation professionnelle et morale: l'information c'est un fait, un fait restitué dans son contexte d'amont et ses perspectives d'aval, à partir desquelles s'expriment des opinions contradictoires, et puis, ensuite, éventuellement, des commentaires. Formation professionnelle et éthique, mais aussi, maintenant, technique.

Comment faire de l'information si on n'a pas un minimum de connaissances dans tous les domaines? Donc formation, inlassablement, des journalistes. Un journaliste a, je crois, un métier comparable à celui de prêtre ou de l'enseignant, qui justifie profondément de la formation. De la formation structurelle, au départ, et de la formation continue, permanente.

Et puis, la formation des consommateurs d'information. Il est terrifiant d'imaginer qu'on ne prépare pas notre population à consommer de la télévision. Et quand on sait qu'aujourd'hui, les enfants passent, en moyenne chaque année, plus de temps devant la télévision que sur les bancs de l'école, il y a là une mission essentielle de préparer à l'examen de la télévision, de préparer à juger, évaluer, critiquer les images, juger, évaluer, critiquer les commentaires. Sinon nous ne sommes plus très éloignés du «meilleur des mondes ».

66 Enfin il y a à être, comme citoyen, attentif et vigilant à toutes les formes de manipulation de l'information ${ }^{31}$."

67 On retrouve, de manière paradoxale, des propos analogues dans un interview de Gustave Thibon:

68 «Il y a une certaine éducation à promouvoir: apprendre aux enfants l'esprit critique, le discernement, leur apprendre que tout ce qui paraît dans les journaux, surtout en période trouble, n'est pas vérité d'Evangile. Pendant la guerre 14-18 les gens disaient: "menteur comme un communiqué»; le peuple savait déjà faire le tri entre le vrai et le faux. Bref, il faut apprendre le sens critique, le minimum de culture, de sagesse, de convivialité, ce qui va permettre de trier dans l'information qu'on reçoit, d'être ouvert et de garder un certain recul. C'est une question de culture ${ }^{32}$.»

69 "Ce qu'on peut faire, c'est choisir les paroles et les images, mentir le moins possible (ce qui est peut-être difficile à tous les gens qui s'occupent de communication : il y a des impératifs politiques, des impératifs économiques. On ne peut tout dire). Et puis, certaines intonations, un certain climat, une ambiance, un halo, quelque chose qui élève l'homme au-dessus de lui-même, qui le fait communier au mystère originel, au mystère dernier. C'est presque impossible à définir. Les moyens sont très réduits, mais enfin, il y a tout de même un accent. Il y a certaines émissions à la télévision, j'en ai vu quelques-unes, où l'on sent la présence de celui qui parle. Elles ont eu, de ce fait, un résultat estimable. Mais il n'y a pas de recette pour être présent, il faut y être, tout simplement ${ }^{33}$. "

D'autres auteurs tiennent un discours auto réflexif sur l'Église et la communication, tel que Bernard PORTE, responsable du groupe Bayard-Presse qui appelle de ses voeux une sorte d'autocritique ecclésiale en s'appuyant sur des textes du P. GABEL d'une part, et du pape Pie XII de l'autre :

71 «E. Gabel écrivait :

72 «Parce que l'Église veut:

73 - un peuple responsable, elle développe l'information,

74 - un peuple libre, elle crée les conditions d'une véritable opinion publique 
75 - un peuple se renouvelant, elle acceptera la critique. Sans critique, l'Église ne peut réaliser cette réforme permanente à laquelle elle est appelée par le Christ et dont elle a besoin en tant qu'institution humaine et terrestre.

Elle doit accepter un jugement collectif dans le peuple chrétien. Pie XII, parlant de l'Église disait :

«Elle est un corps vivant et il manquerait quelque chose à sa vie, si l'opinion publique lui faisait défaut, dont le blâme retomberait sur les pasteurs et les fidèles; Les pasteurs qui n'auraient ni Permis, ni favorisé les conditions de la formation et de l'expression d'une véritable opinion publique dans l'Église, les fidèles qui n'auraient pas eu le courage de constituer et exprimer cette opinion ${ }^{34}$ ?" contradictoires, le doute universel, et la crédulité sans limites. Pour comprendre ce phénomène et tenter d'y porter remède, il me semble que nous pouvons nous poser trois questions:

81 1. L'un de ces deux virus, le doute universel, n'aurait-il pas engendré l'autre, à savoir la crédulité sans bornes?

2. Ces maladies ne seraient-elles pas dues au rejet de la foi et de la pensée chrétienne par ce qu'on a appelé « la modernité »?

83 Il est en effet paradoxal de penser que si nous en sommes là, c'est parce que, sous prétexte de davantage de rationalité, pensée moderne a abandonné:

84 - d'une part, le réalisme aristotélicien et thomiste pourtant solidement fondé sur l'analyse du réel

85 - et d'autre part, le dogme catholique qui, face aux sectes, peut pourtant se prévaloir d'une apologétique et d'une cohérence autrement rationnelles.

86 3. En corollaire, ne pouvons-nous pas penser que nous avons une responsabilité propre dans les maladies modernes de la communication, dans la mesure où nous calquons un peu trop notre (attitude de communiquant sur celle de nos contemporains déboussolés, en négligeant d'affirmer la rationalité, le réalisme et la cohérence de notre vision chrétienne de l'homme et du monde ? ${ }^{36}$ " François Gondrand conseille pour sa part la relecture d'un "petit livre qui a connu un certain succès de librairie depuis deux mille ans, et qui a pour titre les Actes des Apôtres...

88 Je vous conseille de relire et de méditer cette grande leçon de communication qu'est le discours de Paul sur l'agora d'Athènes. Tous les "trucs » y sont - je veux parler des procédés rhétoriques et "dialectiques » que l'on enseigne dans les bonnes écoles et les bons stages de communication - mais avec, en plus, une absence totale de concession.

89 C'est un modèle de discours apologétique que, j'en suis sur, M. le cardinal Poupard a dî souvent méditer dans sa patiente action en faveur du dialogue avec les non-croyants.

90 C'est un modèle de sain dialogue, car il allie les deux pôles, apparemment inconciliables, de toute tentative de ce genre: l'ouverture aux autres, la prise en compte de leur culture et de leurs attentes, et la fermeté la plus absolue quant au contenu de la foi.

91 Ce discours aux Athéniens est à tel point exempt de démagogie qu'il semble tourner court, puisque la plupart des auditeurs s'éloignent et que saint Paul doit partir sous les quolibets. 
92 Et pourtant il s'agit là d'un exercice de communication réussi, Puisque les Actes nous apprennent que quelques hommes et quelques femmes sont quand même restés, ce qui veut dire qu' ils avaient été touchés jusqu'au plus profond d'eux-mêmes ${ }^{37}$."

Comme il en avait l'habitude, André Frossard a fait cavalier seul »... Son propos est conforme à son caractère : Passionné, enthousiaste, voire exalté dès lors qu'il a « la bride sur le cou »... Jugez-en :

"Communiquer", un mot qui a déjà ses titres de noblesse Puisque Bossuet définissait ainsi le mission de l'Église : " enseigner, répandre et communiquer Jésus-Christ 》

Communiquer, c'est donner. Donner quoi? Jésus-Christ Cela tombe bien puisque Jésus dit de luimême qu'il est la

Vérité, de sorte que c'est Lui qu'il faut communiquer, sa personne qui tient lieu de Vérité à tout le monde.

97 Où il est, il y a vérité; là où il n'est pas, il n'y a pas de vérité. Cela simplifie bien les choses pour nous chrétiens ${ }^{38}$.»

98 Quand il veut tordre le cou au fameux concept d'objectivité, il met en avant un argument tout-à-fait inhabituel!

99 "Enfin, il faut être objectif! Un mot qui fait rire dans uni assemblée politique ou journalistique. L'objectivité est impossible, c'est un lieu commun établi, un dogme, il n'y a pas d'objectivité, dit-on. Je prétends que si ! Naturellement, le modèle de toute objectivité est Dieu lui-même ${ }^{39}$. »

100 Il veut rendre à tous le «sens du mystère » et, comptez sur lui, de cela il ne fait pas mystère :

101 "Certains chrétiens se sont dit : les mystères, c'est propre à notre foi, mais ce n'est pas un objet de communication. Ils ont renoncé à parler de dogme, de mystère, d'objet de foi, pour trouver avec les autres des points communs qu'ils ont trouvé dans la sociologie, l'histoire ou la morale.

On en est toujours là. On fait l'impasse sur tout ce qui forme le fond réel de la foi chrétienne, précisément sur le mystère, le merveilleux.

Cette attitude est catastrophique parce que les gens vont chercher l'énigme, le mystère, ailleurs que dans l'Église. Ils se réfugient dans les sectes, chez les voyantes, où ils sont volés comme au coin d'un bois. Ils se réfugient n'importe où, car ils en ont besoin. C'est notre faute, à nous chrétiens, qui n'osons plus affirmer notre objet de foi, notre credo, même si on le chante encore (mais pas toujours) le dimanche ${ }^{40}$. ."

Quant à la question de mettre la communication au service de la «nouvelle évangélisation ", comme le demande le pape, il réagit en journaliste-reporter :

"L'Évangile, comme son nom l'indique, c'est d'abord une "nouvelle", celle de l'Incarnation. La nouvelle, c'est Dieu fait homme. Dieu s'est décidé à apprendre à être homme pendant quelque temps. Nouvelle fantastique!

À l'inverse, si c'est un homme qui, peu à peu, comme le disent aujourd'hui certains manuels, a pris conscience d'une situation privilégiée avec Dieu, cela n'a plus grand intérêt; il n'y a plus de bonne nouvelle, car il n'y a plus de nouvelle du tout. C'est dramatique.

Bien sûr la bonne nouvelle, au sens où on l'entendait autrefois, l'Incarnation, c'est un mystère et dans l'Église catholique, ce sont les mystères qui font les fêtes ${ }^{41}$ ».

Quel contraste avec les prudences avisées de J.-M. Domenach sur le même sujet :

"Vous me parlez de la nouvelle évangélisation? Je ne suis Pas spécialiste de l'évangélisation.

Je me méfie un peu. D'ailleurs même si nous pouvons nous servir de ces moyens de masse 
pour faire comprendre qui nous sommes, pour amener Plus de monde à retrouver les sources chrétiennes nous ne savons pas bien comment le faire. J'ai assez l'habitude de la Parole, sans avoir jamais prêché du haut d'une chaire, pour savoir que nous communiquons dans les marges et que ceux que j'ai pu toucher, modestement, à ma place, et dont j'ai pu parfois orienter la vie dans une direction ou dans une autre, ce n'est pas parce que j'ai voulu le faire. Ils ont entendu telle ou telle parole, ou tel ou tel récit, d'une façon que je ne prévoyais pas ${ }^{42}$ ».

Quant au cardinal Poupart, il s'exprime à la fois comme le pape et le Syndicat National des Journalistes réunis!

«L'exercice correct du droit à l'information suppose que les nouvelles communiquées soient exactes et, autant que faire se peut. exhaustives: vaste programme! Jean-Paul II le dit clairement: " votre profession même vous invite à réfléchir sur cette obligation à l'égard de la vérité et son caractère exhaustif" (Los Angeles, 15 septembre 1987). La vérité de l'information ne se réduit pas à l'objectivité. Recherche du sens, elle suppose le passage de la perception à la réflexion. Les médias donnent à «voir » et à " entendre », d'une manière ou d'une autre. Leur service est de fournir une information fondée sur la vérité, en se gardant de l'altérer sous l'effet de pressions ou de préjugés ${ }^{43}$ "

110 Avec un sens pratique aigu, le prélat nous dévoile quelques aspects de la stratégie du Vatican :

111 «La Salle de Presse du Saint-Siège est devenue un lieu de communication important. Voici déjà un quart de siècle, j'y présentais l'Encyclique "Populorum Progression» de Paul VI à la presse internationale.

112 Jean-Paul II inscrit cette présence institutionnelle dans la réforme de la Curie romaine. Il demande au Conseil pontifical des Communications Sociales de susciter et soutenir l'action de l'Église dans les diverses formes de communication, pour que journaux, périodiques, cinéma et émissions radiotélévisées soient toujours plus pénétrés d'esprit humain et chrétien, et que les moyens de communication catholiques correspondent à leur caractère et à leur fonction propres (Constitution apostolique "Pastor Bonus", 28 juin 1988, art. 169-170). Dans la perspective de la nouvelle évangélisation, le Saint-Siège a acquis l'attribution de droits pour transmettre ses émissions de radio et de télévision par satellite. La nouveauté significative apportée par Jean-Paul II correspond à la ligne directrice de son pontificat: inculture l'Evangile et évangéliser les cultures, un usage qualifié de la communication permet de faire pénétrer le message chrétien dans l'intimité des familles et au cour des sociétés ${ }^{44} »$.

113 Néanmoins, il tient des propos qui se veulent rassurants en citant le pape Jean-Paul II :

(...) « «la vérité ne s’impose que par elle-même, cela suppose que tous doivent respecter la conscience de chacun et de ne pas essayer d'imposer à quiconque sa propre "vérité", restant sauf le droit de la proposer sans pour autant mépriser celui qui pense autrement». La vérité se manifeste à nous aujourd'hui, dans une culture et une société où le refus de Dieu et l'aveuglement moral sont monnaie courante. Devant le laminage des valeurs et des références spirituelles, votre responsabilité inaliénable est extrême $e^{45}$ ".

114 Son message final se veut quand même un encouragement à ses interlocuteurs de la presse :

(...) vous avez "montré une conscience aiguë de la déontologie et de l'éthique de votre profession, je me réjouis de votre volonté de prendre en charge vos responsabilités de chrétiens en Professionnels de la communication ${ }^{46} "$

(...) « Mon message, c'est celui de Jean-Paul II aux « commutateurs » : "l'Église souhaite que vous sachiez qu'elle est à vos côtés. Elle est prête à vous aider de ses encouragements et à vous soutenir dans vos projets positifs. Elle vous offre à la fois un défi et des éloges. " Je prie pour que vous acceptiez cette aide et que vous n'ayez jamais peur de l'accepter. Nous pouvons partager Un espoir commun, enraciné dans une vision de la race humaine harmonieusement unie par la communication. Mesdames et Messieurs, en tant que 
"communicateurs, de la parole humaine, Vous êtes les intendants et les administrateurs d'un immense Pouvoir spirituel qui appartient au patrimoine de l'humanité et est destiné à enrichir l'ensemble de la communauté humaine. Le défi qui vous est proposé exige véritablement générosité, service et amour."

\section{Une pensée laïque encore balbutiante}

119 Face à ce foisonnement des opinions, prises de positions, déclarations issues des milieux catholiques, qu'en est-il de la réflexion éthique en matière d'information et de communication de ceux qui, ne se référant à aucun dogme particulier, qu'ils soient par ailleurs croyants ou incroyants, constatent chaque jour les distorsions, errements, manipulations ou autres malhonnêtetés?

Certes la «laïcisation» de nos sociétés contemporaines pourrait laisser croire que l'ensemble des réflexions sur ces sujets, qui n'émanent pas de milieux croyants, pratiquants, militants, etc. des diverses confessions religieuses, esquisse le territoire d'une pensée laïque relative à ces questions. Tel n'est pas, à mes yeux, le cas.

Car l'absence de références dogmatiques dans les publications, écrits ou discours multipliés ces dernières années ne signifie pas forcément qu'elles aient disparu de la pensée sous-jacente, de l'idéologie implicite de ceux qui les émettent. Et, de plus, les dogmes religieux ne sont plus seuls en cause en ces temps où le culte de l'argent, la religion du profit, de l'ego, etc., constituent autant de manifestations d'une sacralisation des valeurs matérielles et se substituent, dans l'intimité des consciences, aux valeurs spirituelles traditionnelles, religieuses ou non. Un seul exemple : le culte du record qui n'incite pas seulement au dépassement de l'homme par lui-même, mais conduit aussi au défi inconsidéré, au pari stupide, au gigantisme des équipements, installations ou autres œuvres humaines, sans s'attarder au « Toujours plus « d'un célèbre journaliste français.

Le long texte de l'homme d'Église responsable parle moins de Dieu ou d'Église que de l'homme, de l'humain, de l'humanité, de techniques de communication...ou de culture.

La réflexion issue de ces milieux laïques me semble, à l'analyse, tout juste embryonnaire et face à celle des Églises, quelque peu indigente, surtout depuis quelques années. Les penseurs qui fondèrent, au XVIII ${ }^{\mathrm{e}}$ et au XIX ${ }^{\mathrm{e}}$ siècles, l'idée laïque en France trouveraient, en cette fin de $\mathrm{XX}^{\mathrm{e}}$ siècle, la réflexion de ceux qui leur ont accédé et, parfois même se réclament d'eux, bien fade. La «morale naturelle » qui, par un lent travail de maturation a débouché sur la «morale laïque » ne serait-elle plus qu'une survivance spécifiquement belge, défendue par le professeur Gilbert HOTTOIS et ses collègues de 1 Université Libre de Bruxelles? 
Peut-on à ce propos évoquer Massol, fondateur en 1865 de la revue «La Morale Indépendante » qui ne cessa d'affirmer que «la morale se suffit à elle-même et possède une vérité démonstrative trop grande pour qu'on l'affaiblisse en la lisant reposer sur des hypothèses métaphysiques ${ }^{48} "$

"Il est une loi par excellence, écrit-il, conforme à la raison, inscrite dans les coeurs, dont la voix nous dicte nos droits et nos devoirs, dont les menaces nous détournent du mal. »C'est la loi morale. Ce n'est pas une loi dérivée, car elle repose sur un fait avéré, indéniable. "Ce fait, c'est que l'homme est un être libre et responsable, c'est-à-dire une personne, ou du moins qu'il se conçoit comme tel. Que comme tel tout être humain se révolte contre toute contrainte, toute violence, sous quelque forme que ce soit. De là le sentiment de sa dignité, du respect qu'il se porte "lui-même. Mais ce respect de soi, l'homme en présence de l'homme l'exige pour sa personne. Par cela même, il sent forcément que ce même respect est exigible pour tous les autres, dû aux autres. Telle est l'origine du droit et du devoir, qui n'est que le droit reconnu en autrui ${ }^{49}$ "

Trente ans plus tard, en 1895, le savant chimiste (et un Peu philosophe) Marcelin Berthelot écrivit un article sur la relation entre la science et la morale. Il eut un grand retentissement :

"On soutient que la morale échappe à la méthode scientifique, nous avons deux sources de connaissances : la sensation nous fait connaître le monde extérieur et nous montre la subordination de l'individu dans l'humanité, de l'humanité dans l'univers; la conscience nous révèle l'homme seul. Voilà les deux sources de la morale; elle ne vient pas des religions, ce sont elles qui l'ont prise comme fondement. L'histoire de l'humanité prouve qu'entre la morale et le mysticisme il n'existe aucune relation nécessaire; l'homme trouve en lui-même la morale puis l'attribue à la divinité. C'est ce qui a longtemps gêné le progrès de la morale, figée dans des moules dogmatiques: « de là a procédé l'esprit d'intolérance, naturel aux gens qui croient posséder le bien et la vérité absolus et qui, redoutant d'être ébranlés dans leur foi par la critique, veulent interdire aux autres le droit même de la discuter.» Un grand progrès heureusement s'est accompli depuis la Révolution: la morale, comme la science dont elle dérive, est devenue laïque. C'est la morale des honnêtes gens, celle qui proclame le devoir, la vertu, l'honneur, le sacrifice, le dévouement au bien et à la patrie, l'amour des hommes, la solidarité. En outre, de même qu'il y a une science idéale qui inspire la science positive, il y a une morale idéale, celle qui préconise la fraternité des peuples et la solidarité universelle des individus. Cette morale laïque doit pénétrer dans l'éducation; il faut asseoir nos préceptes sur la connaissance des lois exactes du monde intérieur et extérieur ${ }^{50} \%$.

C'est Ernest Lavisse qui donna en 1902, dans le premier numéro des « Annales de la Jeunesse laïque », cette remarquable définition du mot « laïque »:

128 «Etre laïque, ce n'est pas limiter à l'horizon visible la pensée humaine, ni interdire à l'homme le rêve, et la perpétuelle recherche de Dieu : c'est revendiquer pour la vie présente l'effort du devoir.

Ce n'est pas vouloir violenter, ce n'est pas mépriser les consciences encore détenues dans le charme des vieilles croyances; c'est refuser aux religions qui passent le droit de gouverner l'humanité qui dure.

Ce n'est point haïr telle ou telle église ou toutes les églises ensemble; c'est combattre l'esprit de haine qui souffle des religions, et qui fut cause de tant de violences, de tueries et de ruines.

131 Etre laïque, c'est ne point consentir la soumission de la raison au dogme immuable, ni l'abdication de l'esprit humain devant l'incompréhensible ; c'est ne prendre son parti d'aucune ignorance.

C'est croire que la vie vaut la peine d'être vécue, aimer cette vie, refuser la définition de la terre "vallée de larmes", ne pas admettre que les larmes soient nécessaires et bienfaisantes, ni que la souffrance soit providentielle; c'est ne prendre son parti d'aucune misère... 
Etre laïque, c'est avoir trois vertus : la charité, c'est-à-dire l'amour des hommes; l'espérance, c'està-dire le sentiment bienfaisant qu'un jour viendra, dans la postérité lointaine, où se réaliseront les rêves de justice, de paix et de bonheur, que faisaient, en regardant le ciel, les lointains ancêtres; la foi, c'est-à-dire la volonté de croire à la victorieuse réalité de l'effort perpétuel. » (Annales, $n^{\circ} 1$, juin 1902)

Les Jeunesses laïques adoptèrent à l'unanimité l'ordre du jour qui suit :

«La morale laïque doit être scientifique, sociale, humaine. Elle s'appuie sur la raison et sur l'expérience: 1/pour garantir et développer la liberté individuelle; 2 / pour assurer la justice sociale par la solidarité nationale et internationale des individus et des peuples ${ }^{51}$ ”

"Les représentants du haut enseignement philosophique sont tous d'accord pour revendiquer les droits de la raison et la libre recherche de la vérité ", rappelle en résumé Georges Weill ${ }^{52}$.

Mais certains s'interrogeant sur la morale découlant de Ces principes, Félix Pecaut tint à préciser :

"Cette morale laïque est-elle dépourvue, comme on le prétend, d'idées qui puissent parler à l'âme et diriger la conduite des hommes? C'est une erreur. Elle renferme l'idée du droit et du devoir social, la dignité de la personne humaine, l'intelligence des lois naturelles; elle affirme que l'homme est appelé à construire sa destinée librement, puis à réaliser l'idéal qu'il a conçu. Le principe de responsabilité personnelle tient la place d'honneur dans notre éducation; la notion de solidarité vient le compléter ${ }^{53}$ ".

Ces rappels ne sont là que pour montrer combien ces principes sont toujours actuels et pertinents pour aborder le problème moral qui se pose dans le champ de l'information et de la communication. Quelles sont les réflexions actuelles de ceux qui, dans notre pays, pensent ces questions en «laïques»? Examinons pour commencer celle de Jean-Robert Ragache du Grand Orient de France :

139 «La surinformation dont nous souffrons, l'hypertrophie médiatique conduit notre société à la confusion des idées et à l'amnésie. Le temps lui-même n'est plus appréhendé correctement. Des faits révélateurs sont occultés, sciemment ou non, des événements même importants retournent vite à l'oubli. Ainsi notre connaissance du monde est-elle fragmentée, éclatée et nous avons le sentiment de n'avoir aucune compréhension possible de sa marche.» (...) «Du commérage villageois à la fausse nouvelle propagée médiatiquement (rappelons-nous l'épisode de Timisoara) notre société est traversée de ces nouvelles fugitives, amplifiées, déformées, nées d'une imagination trop souvent nocive.» (...)

140 "Dans le monde politique comme dans celui des arts, à la Bourse des valeurs comme dans les relations internationales, nombreux sont les exemples de machination, causes principales des malformations de l'esprit et de l'exclusion de l'autre. Quel contraste avec cette recherche de la vérité qui est une construction raisonnée fondée sur des réalités confirmées et non sur des certitudes insinuées! Quel refus du respect de la dignité humaine, qui est connaissance et reconnaissance! La liberté de pensée, l'examen critique, le refus du conformisme, voilà des armes contre ce qui continue à être l'une des tares d'une société fragilisée.

141 Anéantir le mensonge et l'erreur? Utopie. En tout cas les contraindre et les limiter par la raison et la tolérance ${ }^{54}$ ».

142 Le même représentant de cette organisation philosophique, s'exprimant devant une vaste assemblée, notait également :

143 «La mondialisation de la communication a contribué à démocratiser cette histoire jusque-là à une élite des pays industrialisés. En même temps, les médias hypertrophient l'événement présent et le rendent agressif et monstrueux. Certes l'absence d'information c'est le sommeil de l'esprit ou 
l'explosion d'une imagination débridée. Mais, aujourd'hui, la production médiatique est devenue spectacle, et spectacle totalitaire puisque projeté dans notre vie privée sans nous laisser le temps du jugement et de la critique(...) L'année écoulée nous a montré le temple de cette perversion de l'information qui nous transforme en voyeurs avides et s'efforce de créer un événement quotidien, transformant la réalité en artificialité5 ${ }^{5}$.

Le nouveau président de l'Agence France-Presse (A.FP.), homme important par l'étendue de ses responsabilités, Jean MIOT, a publié il y a quelques années un article intéressant intitulé : «Le développement de la commutation est inversement proportionnel à celui de l'éthique ${ }^{56}$ ».farouche défenseur de l'écrit face à l'hypertrophie des médias audiovisuels, J. MIOT dénonce la "confusion entre " communication et information » qui (...) détourne le journaliste de sa vocation essentielle: informer librement en respectant la vérité et les droits fondamentaux de la personne humaine. Difficile équilibre. C'est au journaliste, et à lui seul, qu'il revient de déterminer les limites de sa liberté d'expression. C'est là qu'intervient l'arbitrage de l'éthique.

145 Toute liberté suppose une responsabilité équivalente. (...) Ce qui distingue la communication de l'information, c'est justement cette référence à la liberté d'expression. (...)

146 Son analyse le conduit à cette assertion explicite :

147 « LE JOURNALISTE S'ADRESSE AU CITOYEN, LE COMMUNICATEUR AU CONSOMMATEUR » l'intérieur. Il est encore temps de réagir si nous ne voulons pas que l'excès de communication ne tue toute déontologie. Cela passe:

153 - par la redéfinition du statut du journaliste dans la Cité;

154 - par une meilleure formation du journaliste " responsabilisé »;

155 - par l'étude d'un code de bonne conduite.»

156 Il y ajoute : «l'éducation du lecteur » et donc "l'apprentissage de la presse à l'école : apprenons à nos enfants à cultiver leur esprit critique par une lecture pluraliste de la presse."

157 Et il conclut: "Non, le développement de la communication ne doit pas se traduire par un apauvrissement de l'éthique. (...) 
débat constamment ouvert et de l'entretenir par la réflexion et par la pratique. Car, comme la communication, l'éthique est un comportement ${ }^{57}$ ”.

Gilbert HOTTOIS fait cette proposition :

"Promotion d'un ensemble de valeurs éthiques typiquement occidentales: il conviendrait de donner une information spécifique sur l'éthique: la notion de valeur, le problème moral, les grandes conceptions éthiques (ici, encore une fois, la dimension historique est indispensable), l'identité du moral par rapport au Politique, au scientifique, à l'esthétique ${ }^{58}$ "

Claude-Jean Bertrand a édité un important dossier intitulé "Télévision et déontologie ${ }^{59}$ " d'une riche substance, introduit dans notre pays le concept d'origine anglo-saxonne de Moyens non-gouvernementaux d'Assurer la Responsabilité Sociale des médias, - les $\mathrm{M}^{*} \mathrm{~A}^{*} \mathrm{R}^{*} \mathrm{~S}$ - et a proposé en 1991 "Quinze moyens d'améliorer les médias ${ }^{60}$ ».

Daniel Bougnoux, sans aborder le débat en termes d'éthique, l'a cependant enrichi par son récent article "La communication contre l'information ${ }^{61}$ ", qui conclut, sur le mode du philosophe, dans le même sens que Jean Miotl'avait fait en homme de presse.

Enfin Guattari, dans un article intitulé : «Pour une éthique des médias ${ }^{62}$ », fait à partir des constatations relevées dans le traitement de l'information à la télévision un certain nombre de propositions :

« En France, les télévisions ne sont plus sous la tutelle directe des instances étatiques et politiques. Mais, à l'intérieur de leur chaîne, les journalistes demeurent assujettis à leur direction et très souvent pratiquent une autocensure, une soumission à l'opinion régnante.. Il y a beaucoup à faire pour aboutir à une véritable démocratisation de l'information qui passe par la mystification du mythe de V» information objective ». Les faits, auquels'ils soient, sont sujets aux interprétations les plus diverses. »(...)

"A ce niveau, il semble nécessaire d'instituer une sorte d'équivalent des pouvoirs judiciaires. Des commissions nationales et internationales d'éthique devraient garantir que les abus en la matière pourraient être, sinon sanctionnés, du moins Portés à l'attention du public.» (...) Toute une éducation du public est à faire ou à refaire. Il en va de l'avenir des institutions démocratiques. » (...)

"Il n'y a pas de destin en la matière, de causalité linéaire, d'entropie incontournable. Des moyens nouveaux sont à portée de main. Mais seule une volonté collective, l'affirmation de nouvelles finalités sociales, éthiques et culturelles, associées à une multitude d'initiatives, d'expérimentations, pourront s'en saisir.» (...)

69 La question de l'éthique des médias et de l'orientation prospective des nouvelles technologies de communication, d'intelligence artificielle et de commande constitue, avec la problématique écologique, un des deux axes de recomposition d'une pensée de progrès pour la planète d'aujourd'hui."

\section{Retour à une morale non-dogmatique}

Sans entrer dans le débat soulevé par Emmanuel KANT à propos du paradoxe de Benjamin Constant ${ }^{63}$ sur le droit de mentir, notons cependant l'importante distinction qu'il fait entre vérité, véracité et loyauté: "C'est donc un commandement de la raison qui est sacré, absolument impératif, qui ne peut être limité par aucune convenance : en toute déclaration, il faut être véridique ${ }^{64}{ }^{\Perp}$.

Que n'enseigne-t-on Kant dans les formations à la communication? 


\section{NOTES}

1. ETCHEGOYEN A., La valse des éthiques, F. Bourin, Paris, 1991, pp. 167-193.

2. ETCHEGOYEN A., idem., p. 171.

3. ETCHEGOYEN A., ibid., p. 173.

4. Communication, vol. 13, n 1, printemps 1992, université Laval, Ste Foy, Québec.

5. Cahiers de Recherche Éthique, Éd. Fides, Québec, trimestriels depuis 1988.

6. In Le Journaliste, SNJ, Paris, $4^{\mathrm{e}}$ trimestre 1991, p. 12

7. Idem. 
8. Ibid.

9. Pinto de Oliveira, Éthique de la Communication sociale, vers un ordre humain de l'information et de la communication dans le monde, Éditions universitaires, Fribourg, 1987.

10. Les actes de ce Colloque ont été édités sous le titre: Éthique et communication, Beauchesne, Paris, 1991.

11. Voir référence précédente, pp. 16-17.

12. Idem., p. 33.

13. Ibid.,p. 38.

14. Ibid., p. 79.

15. Ibid., p. 81.

16. Ibid., pp. 82-83.

17. Ibid., pp. 83-84.

18. Ibid., p. 84.

19. Voir référence $8, \mathrm{pp} .116-119$.

20. Idem. p. 53.

21. Ibid., pp. 54-55.

22. Ibid., p. 55.

23. Ibid., p. 101.

24. Ibid., p. 102.

25. Voir référence 9, p. 66

26. Idem., p. 67.

27. Ibid., p. 68.

28. Ibid., p. 69.

29. Voir référence 8, p. 19.

30. Idem., p. 21.

31. Voir référence 8, pp. 21-23.

32. Idem., p. 40.

33. Idem., p. 40.

34. Ibid., p. 96.

35. Ibid., p. 98.

36. Idem., pp. 127-128.

37. Voir référence $8, \mathrm{pp} .84-85$.

38. Ibid., p. 27.

39. Ibid., p. 28.

40. voir référence p. 31

41. Idem., p. 29

42. Ibid., p. 57.

43. Ibid., p. 136.

44. Voir référence 8, p. 138.

45. Idem, p. 51.

46. Idem, p. 151.

47. Ibid., p. 152

48. MASSOL in La Morale Indépendante, Paris, $\mathrm{n}^{\circ}$ 1, 6 août 1865.

49. MASSOL, cité par WEILL G., Histoire de l'idée laïque en France, Lib. Félix Alcan, Paris, 1925, pp. 200-201.

50. BERTHELOT M., cité par WEILL G., op.cit., pp. 306-307.

51. Cité in WEILL, G., pp. 318-319.

52. WEILL G., op.cit., p. 329.

53. Idem, p. 337.

54. In Humanisme, Paris, $\mathrm{n}^{\circ}$ 201-202, décembre 1991, p. 11 
55. Idem., p. 91

56. MIOT J., Le développement de la communication est inversement proportionnel à celui de l'éthique ", in Communications et Langages, $n^{\circ}$ 89, Retz, Paris, $3^{\mathrm{e}}$ trimestre 1991, pp. 95-105.

57. HOTIER H., L'éthique de la communication, in Le Monde, novembre 1990

58. HOTTOIS G., Philosophie, morale et société, U.L.B., Bruxelles, 1982, p. 92, voir également les pp. 84-87 sur l'information.

59. BERTRAND C.-J., Télévision et déontologie, Dossiers de l'audiovisuel n 36, INA-Documentation Française, Paris, mars-avril 1991

60. BERTRAND C.-J., in Médias Pouvoirs $\mathrm{n}^{\circ}$ 21, Bayard-Presse, Paris, 1er trimestre 1991,

pp. 115-122.

61. BOUGNOUX D., in Études, Paris, mars 1992.

62. GUATTARI F., Pour une éthique des médias, in Le Monde, Paris, 6 nov. 1991.

63. KANT E., Sur un prétendu droit de mentir par humanité(1797), in Théorie et pratique. Librairie philosophique, J. VRIN, Paris.

64. KANT E., op.cit., pp. 69-70.

65. In Éditorial, Revue Entreprise Ethique, n 3, octobre 1995, p. 4.

\section{RÉSUMÉS}

En réponse au thème "l'information au service de la communication », et réticent à ce principe de vassalisation de l'une à l'autre, l'auteur vise à l'inverse leur rapprochement en mettant en évidence ce qui les rassemble et les réunit ; une commune exigence éthique. Passant en revue des travaux anciens ou récents, abordant cette question, l'éthique de l'information et de la communication, il met en relief : 1 - la naïveté de nombreux discours déontologiques issus des milieux professionnels de l'information et de la communication. 2 - l'importance de la réflexion critique inspirée par certains secteurs de l'Église catholique en dépit des attitudes dogmatiques de son pontife et de la Curie. 3 - l'insuffisance d'une réflexion indépendante de toute référence dogmatique, encore balbutiante malgré l'apparente laïcisation des pratiques de larges pans des sociétés contemporaines. Il plaide pour l'approfondissement et l'enrichissement d'une pensée authentiquement laïque, proposée à tous les acteurs, sans distinction, de l'univers de l'information et de la communication.

In response to the thème "Information at the service of communication ", and being opposed to this principle of subordination of the one to the other, the author has tried conversely to bring them together by highlighting what brings them together and what unites them, that is to say joint ethical demands. He reviews work that has been done on this theme in the past and also more recently and tacckles the question of the ethics of information and communication by pointing out the following: 1 - the naivety of many deontological pronouncements made in the professional fields of information and communication. 2 - the importance of critical reflection inspired by some sectors of the Catholic Church despite the dogmatic attitudes of the pontiff and the Curia. 3 - the insufficiency of the reflection independent of all dogmatic reference. This thought is still hesitant despite the apparent laity of sectors in contemporary societies. He pleads in favour of the deepening and the enriching of an authentic lay philosophy which would be proposed without discrimination to all those in the world of information and communication. 


\section{AUTEUR}

\section{JEAN DEVÈZE}

Jean Devèze, professeur en sciences de l'information et de la communication à l'Université de Marne-la-Vallée. près de Paris, dirige le DESS de «Communication des entreprises, des administrations et des institutions ». Ses travaux portent sur de nombreux domaines de ce champ scientifique. Les questions relatives à l'éthique et aux pratiques professionnelles des acteurs de l'information et de la communication sont au cœur de ses interrogations. 